\title{
Introduction.
}

\section{Les enjeux de la conférence de Paris. Penser autrement la question climatique}

\author{
Catherine Aubertin, Michel Damian, Michel Magny, Claude Millier, Jacques Theys, \\ Sébastien Treyer
}

Les contributions de ce dossier sont centrées sur les négociations qui se dérouleront à Paris en décembre 2015 lors de la COP $21^{1}$. Elles questionnent les représentations qui ont conduit au cadrage initial de la question climatique et invitent à décaler notre regard, à changer nos approches pour permettre d'explorer de nouveaux chemins face aux enjeux du changement climatique.

La Convention-cadre des Nations unies sur le changement climatique a construit le réchauffement climatique comme un problème de pollution, une « externalité » comptabilisée au moyen d'une unité unique équivalant à une tonne d'émissions de $\mathrm{CO}_{2}$, que les mécanismes de marché pouvaient réduire à moindre coût. Un accord multilatéral global portant sur des objectifs de réduction devait s'imposer à tous les États, tout en tenant compte des « responsabilités communes mais différenciées ".

Ce cadre a montré ses limites et, vingt-trois ans après la signature de la Convention, les émissions de gaz à effet de serre n'ont jamais été aussi importantes, alors que le décalage est patent entre, d'un côté, la production de faits scientifiques et les recommandations du GIEC et, de l'autre, l'enlisement des négociations, les actions des États et la prise en compte par les sociétés de la question climatique. La construction progressive d'une forme de confiance entre États, préalable à l'action conjointe, ne se fait qu'avec une lenteur qui contraste très fortement avec l'impératif d'urgence souligné par le GIEC ; les politiques climatiques des gouvernements sont de plus en plus nombreuses au Nord comme au Sud, mais elles semblent encore bien timides; beaucoup d'actions collectives ou à l'échelle locale montrent l'engagement de plus en plus fréquent de citoyens ou de mouvements

1 Conférence des parties de la Convention-cadre des Nations unies sur les changements climatiques. sociaux pour prendre en charge la question climatique, mais ils ne semblent pas être à l'échelle des défis tels qu'exposés par les scientifiques.

Entre le diagnostic d'urgence d'une action globale coordonnée et la mise en mouvement très lente d'actions hétérogènes se pose la question du message que la science doit porter aujourd'hui pour cadrer les réflexions politiques et stratégiques, au-delà de la mise à l'agenda ; ce qui exige des traductions et des débats de type "sciences en sociétés » appliqués aux caractéristiques très spécifiques du fait climatique. Nous ne sommes plus dans une phase de sensibilisation, mais dans une période où désormais la question essentielle est celle d'une bonne articulation entre la recherche et les orientations ou les moyens de l'action. La revue Natures Sciences Sociétés à la fois par son ambition interdisciplinaire et son attention portée à l'interface sciences/sociétés est un lieu où cette question prend une résonance particulière.

Les auteurs qui ont répondu à notre appel à propositions $^{2}$ font partie de cette communauté de chercheurs en sciences sociales qui s'engagent dans les débats sur le changement climatique, et acceptent de ne pas s'en tenir qu'à une forme d'extériorité critique. Ils sont forces de réflexion pour apporter des éclairages à ces débats, et de propositions pour imaginer un autre cadrage des enjeux, des problèmes et des solutions, une autre relation entre sciences et sociétés. Ils s'interrogent sur ce que le changement climatique, comme objet de négociation, dit de nos sociétés. Ils interprètent comment ses composantes matérielles et objectives s'articulent à ses composantes culturelles, symboliques et subjectives. Le déroulé des négociations, avec ses jeux géopolitiques et la construction des

\footnotetext{
2 NSS s'invite aux débats de la conférence de Paris sur les changements climatiques, Natures Sciences Sociétés, 22, 1, http:/ / www.nss-journal.org/articles/nss/pdf/2014/01/ nss140020.pdf.
}

Auteur correspondant : C. Aubertin, catherine.aubertin@ird.fr 
normes et outils, fournit de nombreux objets de recherche pour les sciences sociales, que cela soit en histoire des sciences, en relations internationales, en économie, en géographie. Les contributions réunies ici rendent compte de quelques-unes des principales analyses critiques de la construction du cadrage du problème climatique : la logique des négociations (Amy Dahan et Hélène Guillemot), les choix des outils économiques (Michel Damian, Mehdi Abbas et Pierre Berthaud; Christophe Cassen, Céline Guivarch et Franck Lecocq), les relations avec la problématique du développement (Sandrine Mathy), l'articulation des échelles globales et locales (Hervé Brédif, François Bertrand et Martine Tabeaud), la montée en puissance de l'adaptation (Guillaume Simonet), la faible prise en compte de la consommation (Ghislain Dubois et Jean-Paul Ceron), les relations Nord-Sud (Moïse Tsayem, Roger Ngoufo et Paul Tchawa). Certes, on peut regretter que des sujets d'importance majeure comme les grandes villes, l'agriculture et les littoraux par exemple, ou des points de vue, comme celui des sciences juridiques, n'aient pas pu figurer dans ce numéro, nécessairement limité, mais la diversité des angles et des objets montre bien l'ampleur des chantiers ouverts sur de nombreux fronts.

On trouvera d'abord dans toutes les contributions une critique $\mathrm{du}$ « modèle linéaire » subordonnant l'action à la connaissance : la science fournit des diagnostics et des faits au politique qui s'appuie sur ces connaissances pour développer des solutions (Dahan et Guillemot; Tsayem et al.). Toutes les analyses montrent au contraire une coproduction des normes et des stratégies, des préconisations politiques de la part des scientifiques, une circulation des savoirs entre les sphères de la recherche et de la décision, et, tant bien que mal, entre l'échelle globale et l'échelle locale. Il s'agit de désenclaver les politiques climatiques en mettant fin à l'exceptionnalisme climatique: le risque climatique considéré comme ne ressemblant à aucun autre et plus menaçant que tous les autres. En l'érigeant en problème environnemental ultime, les négociations ont établi une sorte de mur coupe-feu entre le climat et les autres questions qu'affrontent nos sociétés. Exceptionnelle menace, il aurait à lui seul suffi à changer le cours des autres décisions de nos sociétés. Après le constat de l'incapacité de la conférence de Copenhague à décréter un tel changement, et le retour vers les difficiles arbitrages nationaux pour mettre en place des politiques climatiques, le changement climatique apparaît clairement aujourd'hui comme un problème géopolitique, économique, énergétique, de commerce international, autant qu'un problème environnemental.

Le mode d'articulation entre gouvernance globale, politiques nationales et initiatives locales est donc au cœur de la question climatique. Le modèle top-down où il revient aux seuls États - s'accordant ou non entre eux - de prendre des décisions est bousculé. Il n'est plus question d'un partage du fardeau négocié dans le cadre d'un traité international contraignant: la conférence accueillera les propositions de «contributions nationalement déterminées ", avec des règles pour l'Accord qui sera certainement signé à Paris déjà préemptées, pour l'essentiel, par le G2 États-Unis/Chine (Damian et al.). Un des enjeux majeurs de la conférence de Paris est donc de redéfinir, dans le cadre multilatéral, meilleur garant aujourd'hui d'une légitimité internationale, les règles et principes qui permettront de coordonner ces actions nationales, pour éviter qu'elles soient trop disparates et trop peu ambitieuses. Un phénomène construit comme mondial tel que le changement climatique a du mal à prendre en compte la diversité des situations locales et peine à considérer la dimension socioéconomique globale des initiatives à engager sur le terrain, au-delà du secteur de l'énergie qui a beaucoup focalisél'attention des économistes du climat. Le mécanisme de Réduction des émissions dues à la déforestation et à la dégradation des forêts tropicales (REDD) illustre ainsi le difficile passage d'un outil négocié sur la scène internationale à son application opérationnelle locale dans de délicates relations entre un Nord industriel et un Sud forestier (Tsayem et al.). Lié ou non aux politiques nationales, le niveau local s'organise et les approches territoriales donnent une autre vision de l'expertise et de l'action (Brédif et al.).

Les politiques climatiques doivent aussi trouver de nouvelles formes de légitimation. La vision climatocentrée a en effet placé en haut de la hiérarchie des priorités la réduction des émissions de gaz à effet de serre avant les enjeux du développement, de santé, d'emploi, de sortie de la pauvreté (Mathy). Nous savons pourtant que les politiques qui ont un impact sur les réductions des GES ne peuvent être dissociées d'autres enjeux nationaux, régionaux, locaux et qu'elles nécessiteront des arbitrages avec d'autres enjeux, comme celui des droits des pays à exploiter leurs ressources naturelles, y compris leurs rentes charbonnières ou pétrolières ${ }^{3}$. Le concept de cobénéfices des politiques climatiques connaît ainsi un intérêt croissant, mais les cadres conceptuels, notamment dans les modélisations économiques, peinent encore à représenter cette notion de manière opérationnelle pour les politiques (Cassen et al.).

Ce dossier revient par ailleurs sur la manière dont le thème de l'adaptation, à peine évoqué dans le texte de la Convention et qui reste un concept difficile à cerner, s'est inscrit à l'agenda des négociations et a gagné en force dans les rapports du GIEC, permettant à la fois un décloisonnement disciplinaire et l'expression des pays du Sud (Simonet). Si l'objectif de l'atténuation reste une trajectoire des émissions de GES qui maintiendrait l'augmentation de la température moyenne mondiale

\footnotetext{
3 Voir le Regard de J.-M. Martin dans ce numéro.
} 
en dessous de $2^{\circ} \mathrm{C}$ en 2100 , l'adaptation vise à garantir des moyens d'action collective pour se préparer à un monde à $+2{ }^{\circ} \mathrm{C}$ (mais à plus forte raison aussi à un monde à $+3{ }^{\circ} \mathrm{C},+4{ }^{\circ} \mathrm{C},+5^{\circ} \mathrm{C}$ ?) afin de sécuriser les systèmes humains et répondre aux demandes de justice et d'équité des pays du Sud.

En privilégiant une vision macroéconomique, les politiques climatiques ont enfin souvent négligé la question des modes de vie et de leurs évolutions. L'attention portée aux modes de consommation permettrait de redonner un rôle aux initiatives locales et à la société civile, et de mieux prendre en compte des fuites carbone dans les échanges internationaux. Elle devrait conduire dans les négociations internationales à ne pas faire reposer la solution sur les seuls accords entre États fondés sur des évaluations d'émissions liées à la production, débouchant sur des marchés carbone destinés aux seuls industriels (Dubois, Ceron). Il s'agit aujourd'hui d'impliquer tous les acteurs, ONG, mouvements sociaux, communautés locales, syndicats, etc., dans le cadre de politiques multi-objectifs et de donner une plus grande place aux questions d'innovation élargie, de partenariat technologique, de solidarité, de manière de produire, de consommer.

Il importe en conclusion de sortir de l'impasse d'une représentation du risque climatique focalisé sur les émissions de GES, représentation qui a montré ses limites, d'en finir avec l'exceptionnalisme climatique et le mirage d'un accord universel contraignant, sans que cela soit pour autant un prétexte pour ne pas agir. Avec les préoccupations et inquiétudes relatives au climat s'entremêlent bien d'autres risques environnementaux, d'autres enjeux liés aux questions de " développement », de pauvreté, de sécurité, mais aussi de solidarité et de réconciliations nationales que l'on peine à reconnaître ou imaginer. Il s'agit de reconsidérer dans son ensemble la question climatique, en suivant toutes les pistes qu'elle invite à explorer, en rassemblant les thèmes jusqu'alors dispersés et en redistribuant les cartes parmi les acteurs. Les articles réunis dans ce numéro spécial veulent contribuer à cet « agenda des solutions » que la conférence de Paris cherche à construire. 